\title{
Análisis de la movilidad en campus universitarios integrados en zonas urbanas
}

\author{
Analysis of integrated mobility in college campus in \\ urban areas
}

마맘

Francisco Lucas-García', Jesús Racero-Moreno² ${ }^{2}$ Cristina Torrecillas', José Manuel García-Sánchez²

UNIVERSIDAD DE SEVILLA. Escuela Técnica Superior de Ingeniería. Dpto. de Ingenieria Gráfica y y Dto. de Organización Industrial y Gestión de Empresas². Avda. Camino de los Descubrimientos, s/n - 41092 Sevilla.

\section{DOI: http://dx.doi.org/10.6036/7660|Recibido: 14/05/2015• Aceptado: 26/05/2015}

\section{ABSTRACT}

- Urban transport studies have been characterized by focusing on the analysis of mobility problems between different areas, mainly looking to minimize travel time. In recent years, negative effects (externalities) of urban mobility have become very important because of environmental aspects. Mobility to campus or universities represents a large number of journeys within city. In this study, mobility patterns of students, workers and residents in an integrated urban campus environment are described. An assessment of energy consumption and emissions of pollutants into the atmosphere, caused by these displacements, to have a higher level of awareness of damage caused to environment was proposed.

The paper conclusions are that although there are ways more sustainable, less polluted and more economical transport, people are reluctant to use them. Thereason is that while there are infrastructures that allow use of other more polluted and less sustainable modes due to the cost time, convenience or because people costs to change their habits.

To encourage more sustainable transport, firstly we make awareness people of the pollution and to facilitate, encourage and promote most sustainable modes and secondly to penalize most polluting and less sustainable transport.

- Key Words: mobility, university, energy and environmental inventory.

\section{RESUMEN}

Los estudios de transporte urbano se han caracterizado por centrarse en el análisis de los problemas de movilidad entre diferentes zonas, buscando principalmente la minimización del tiempo de viaje. En los últimos años han cobrado gran importancia los efectos negativos (externalidades) que la movilidad urbana genera, entre ellos los aspectos ambientales. La movilidad con motivo de viaje a campus o centros universitarios supone un gran número de los desplazamientos realizados dentro de la ciudad. En este estudio se describen los patrones de movilidad de los estudiantes, trabajadores y residentes en un campus universitario integrado en un entorno urbano. Se realiza una evaluación del consumo energético y las emisiones de contaminantes emitidas a la atmosfera provocados por estos desplazamientos, y así tener un mayor nivel de concienciación sobre el daño producido al medio ambiente.

De las conclusiones obtenidas en este trabajo se debe destacar que aunque existan modos de transportes más sostenibles, menos contaminaste y más económicos, las personas se resisten a utilizarlos mientras existan infraestructuras que permitan el uso de otros modos más contaminantes y menos sostenibles, ya sea por el tiempo, comodidad o porque les cuesta cambiar de hábitos.

Para poder incentivar estos usos más sostenibles, primero hay que concienciar a las personas de la contaminación que están produciendo e igualmente facilitar, incentivar y potenciar los modos más sostenibles; y por otro lado, penalizar los más contaminantes y menos sostenibles.

Palabras Claves: movilidad, campus universitario, inventario energético y ambiental.

\section{INTRODUCCIÓN}

El transporte es una de las fuentes más importantes de emisiones de gases de efecto invernadero, siendo los transportes por carretera el 80\% de estas emisiones en España [7].

La demanda del transporte tiene una dificultad añadida, ya que los viajes son decisiones individuales de cada persona, con lo cual existe un problema en el que las probabilidades de viajes son aleatorias, aunque se adaptan a determinados patrones.

Tradicionalmente los estudios de movilidad se basaban en un modelo de cuatro etapas (Four Stage Model o FSM) [19]. El FSM es una herramienta para predecir la demanda y el rendimiento de un sistema de transporte, a escala regional o subregional, Fig. (1). Es un modelo general y no incorpora aspectos para la evaluación ambiental o de la sostenibilidad.

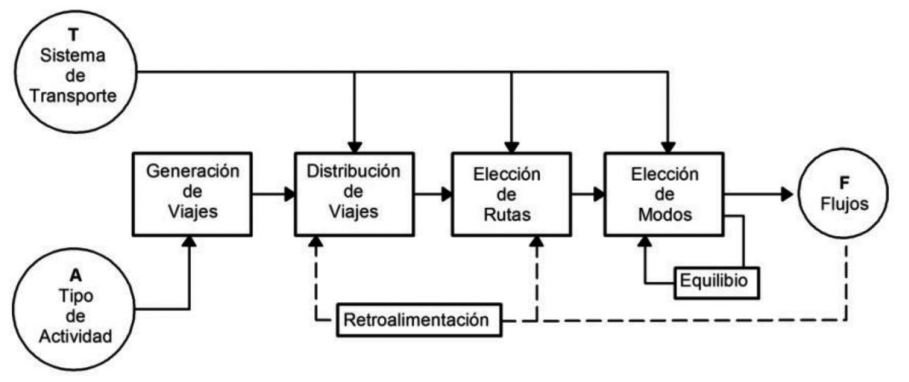

Fig. 1: El modelo de cuatro etapas (FSM)

Algunos gobiernos han desarrollado sus propias guias de transporte, que incluye criterios de SOSTENIBILIDAD. En Francia, por ejemplo, la primera guía de plan de transporte se desarrolló en 1982, pero no fue hasta 2007 cuando se incluyó un análisis sobre la misma [1]. Este plan de sostenibilidad, Ilamado Plan de déplacements Urbains, o PIU [17], es de obligatorio cumplimiento y revisable cada cinco años. En Reino Unido, la guía se llama "Plan de Transporte Local" [16] y es de aplicación en áreas urbanas; es una 
guía dinámica ya que su objetivo cambia según los indicadores de las evaluaciones anteriores. Por último, en otros países como por ejemplo Italia, se describen planes de transportes cuya revisión no se realiza hasta pasados diez años [15]. En España, los planes de transportes o guías son desarrollados por el Instituto para la Diversificación y Ahorro de la Energía (IDAE) y describen una serie de acciones asociadas a la demanda, para desarrollar e implementar estrategias orientadas a cambiar los hábitos de movilidad. Estas estrategias deben ser atractivas para buscar un uso más racional del vehículo privado y fomentar los modos de transporte más sostenibles [8].

Sobre estas guías/planes, las ciudades mayores de 50.000 habitantes en el caso de España, desarrollan sus propios planes locales que por lo general no tienen en cuenta variables medioambientales.

En el caso de planes de transporte a campus universitarios, estos son muy similares a los planes de transportes de trabajadores [8]. Debido a que en estos planes son comparables los viajes de trabajadores y los viajes de estudiantes. Algunos ejemplos de estos planes son los realizados por Robusté [2], Rozas [3], Toor [4] o Colomer [5].

En estos planes se presentan criterios de sostenibilidad pero no contemplan el estudio sobre el consumo de energías o la emisión de contaminantes, aspectos medioambientales que cada día tienen más importancia.

Los trabajos en la literatura sobre de planes de movilidad en centros escolares o campus universitarios no fijan una metodología y se centran en analizar los patrones de movilidad basándose en encuestas, no incluyendo estudios energéticos o medioambientales (Balsas [21], Miralles [22] y Shannon [23]).

El objetivo de este trabajo es realizar el estudio de la movilidad en un campus universitario integrado en un centro urbano, realizando un diagnóstico de las posibles necesidades, y haciendo especial hincapié en la sostenibilidad del mismo. El análisis del presente trabajo se centrará en el estudio de diversos indicadores utilizados tradicionalmente (transporte privado, transporte público, aparcamientos), al que se han incorporado nuevos indicadores asociados a criterios de sostenibilidad (inventarios energéticos, ambientales y modos de transporte sostenible). Estos nuevos indicadores no se han tenido en cuenta hasta ahora en este tipo de planes y deberían ser utilizados como referencia para proponer medidas que mejoren la movilidad y la calidad ambiental.

\section{METODOLOGÍA}

La metodología aplicada se puede establecer en 4 etapas:

Primera etapa. "Recogida de datos"

Esta etapa se estructura en dos fases: la toma de datos de la zona objeto de estudio y realización de encuestas.

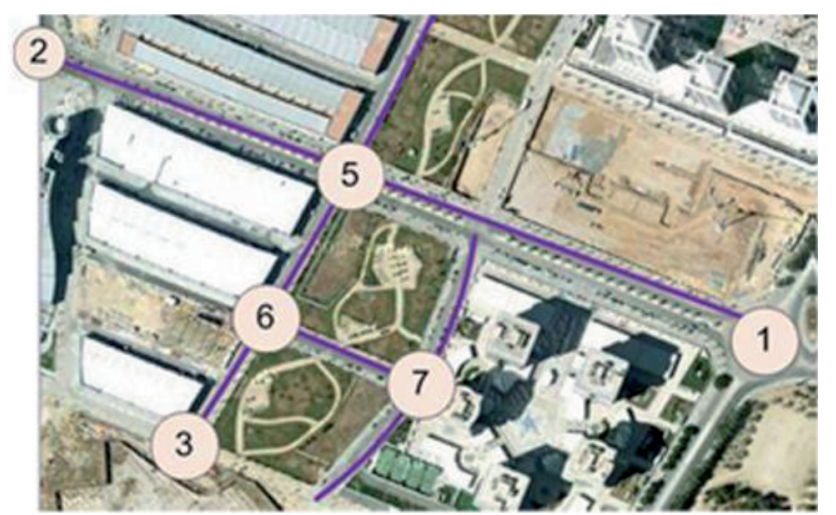

Fig. 2: detalle del grafo superpuesto a una ortofoto de la zona de estudio
La toma de datos: en una base cartográfica se digitalizan las vías urbanas y se realiza una división o zonificación del espacio. Esta digitalización consiste en modelar el viario mediante un grafo dirigido donde los vértices se corresponden con intersecciones de las calles o vías y los arcos o tramos que unen estos vértices son las calles o vías, uno por cada sentido de circulación. Cada vértice es identificado por un número unívoco y un arco por dos vértices o nodo que se denominan origen y destino en función del sentido de circulación [18]. El resultado del proceso de digitalización es un mapa de fondo con un grafo superpuesto, esto es la base de la organización para la toma de datos, ver Fig. (2).

Posteriormente, en fichas prediseñadas, se recogen los datos relativos a la infraestructura de las vías (detalles de estacionamientos, intersecciones, pasos de peatones, vías y estacionamientos de bicicleta) y la información sobre oferta y demanda del transporte público (líneas, paradas y frecuencias).

La realización de encuestas: en éstas se recogen datos como las distancias recorridas, horarios y modos de transportes utilizados, motorización, tipo de combustible, motivos por el que utiliza el vehículo privado, capacidad de compartir coche y disponibilidad a cambiar de modo, así como las causas por las cuales realizarian el cambio.

Para mantener un alto nivel de fiabilidad se establece un número mínimo de encuestas a realizar. La metodología empleada para la realización de las encuestas está basada en el manual de encuestas de movilidad [12] donde, para calcular la muestra sobre el número de encuestas a realizar con un índice de confianza del $95 \%$ así como un error del $10 \%$, se ha tomado la siguiente ecuación Ec. (1):

$$
n=N \cdot \frac{Z_{\alpha}^{2} \cdot p \cdot q}{\left(e^{2} \cdot(N-1)+\left(Z_{\alpha}^{2} \cdot p \cdot q\right)\right)}
$$

\section{Ec. 1: Cálculo muestral para el numero de encuestas.}

Dónde:

$\mathrm{N}$ : Tamaño de la población

$Z_{\alpha}^{2}$ : Coeficiente para el nivel de confianza $(1-\alpha)$

e: precisión deseada para el estudio

p: proporción de la población que cumple un requerimiento determinado. Como este número es indeterminado ya que se pregunta sobre varios supuestos, se establece el valor más desfavorable $p=0.5$

$q: 1-p$, que en este caso $q=0.5$

\section{Segunda etapa. "Análisis"}

Con los datos recogidos en la etapa anterior se analizan los usos y modos de transportes. Su objeto es mostrar el estado actual y ver si son necesarias acciones de mejoras. El análisis se realiza en base al tipo de transporte utilizado, privado o público, en bicicleta, peatonal, los horarios utilizados y la ocupación de los aparcamientos. Esta información se sintetiza mediante indicadores numéricos y gráficos que permiten un análisis visual con mayor concreción.

Tercera etapa. "Inventario energético"

Se realiza un inventario del consumo de combustibles y de emisiones de gases contaminantes al medio ambiente como resultado de los desplazamientos al campus universitario, desagregando los consumos y emisiones por tipo de vehículos [18].

Con este cálculo de consumos de energías y emisión de contaminación se quiere conseguir un doble objetivo, por una parte el crear un nivel de concienciación mayor sobre el daño producido al medio ambiente y por otra, realizar una evaluación del coste energético que suponen estos desplazamientos. 
Para realizar el cálculo de los consumos de combustible hemos utilizado los siguientes datos extraídos de las encuestas:

- Distribución modal: expresa el peso porcentual de los modos de desplazamientos más utilizados (bus, cercanía, vehículo privado, bici o peatonal).

- Distancia media diaria recorrida: de los estudiantes o trabajador en su desplazamiento de ida y vuelta.

- Grado de Ocupación de los vehículos privados: obtenido por las encuestas y también mediante aforos. En el caso de los transportes públicos estos datos son facilitados por las empresas o compañía que lo gestionan.

La expresión utilizada para obtener la emisión de contaminantes para un modo de transporte y tipo de vehículo está basada en la expresión (2), donde se multiplica el número de usuarios, por la distancia media recorrida en ese modo, por el factor de emisión y repartida por el número de ocupantes:

\section{$\sum_{\text {Modo transp. }} \sum_{\text {Tipo veh. }} \frac{\text { Usuarios }_{\text {Modo Veh }} * \text { Distancias }_{\text {modo }} * \text { Factor de Emision }_{\text {Veh }}}{\text { Ocupación }_{\text {Modo }}}$}

\section{Ec. 2: Expresión para el cálculo de los consumos energéticos}

Dónde:

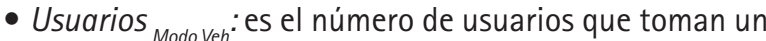
modo de transporte (vehículo privado, autobús, tren) con un tipo de combustible (gasolina, gasoil, gas).

- Distancia modo: es la distancia media de los desplazamientos en ese modo.

- Factor de Emisión veh: es el factor de emisión por kilómetro según el tipo de vehículo.

- Ocupación ${ }_{\text {MоDMd Modo }}$ : es la ocupación media según el tipo de transporte.

Para obtener los factores de emisión de contaminantes utilizamos los índices proporcionados por la Agencia Estatal de Medio Ambiente (AEMA) [24], donde se muestran los niveles de emisión de acuerdo a los kilómetros recorridos, tipo de transporte y combustible. Con respecto al vehículo privado los datos proporcionados por la AEMA suponen equivalente las emisiones de vehículos diésel y gasolina, ver Tabla 1.

\begin{tabular}{|c|c|c|c|c|}
\hline $\begin{array}{c}\text { FACTOR DE EMISIÓN } \\
\text { [gr/pasajero Km] }\end{array}$ & $\begin{array}{c}\text { Vehículo } \\
\text { privado }\end{array}$ & Autobús & $\begin{array}{c}\text { Tren } \\
\text { (diésel) }\end{array}$ & $\begin{array}{c}\text { Tren } \\
\text { (eléctrico) }\end{array}$ \\
\hline C02 & 240 & 70 & 80 & 16 \\
\hline CO & 21 & 1 & 0,01 & 0,001 \\
\hline HC & 2,9 & 0,5 & 0,004 & 0,0004 \\
\hline Nox & 1,5 & 0,9 & 0,3 & 0,05 \\
\hline
\end{tabular}

Tabla 1: Factores de emisión de contaminantes [24]

Cuarta etapa. "Propuestas".

En esta etapa se deben establecer las posibles propuestas de mejoras una vez analizados los puntos anteriores. Si se establecen propuestas, éstas deben ser revisables en periodos de tiempos preestablecidos.

Seguidamente se realiza la aplicación de estas cuatro etapas a un campus universitario integrado en la ciudad de Sevilla.

\section{LOCALIZACIÓN Y DATOS DE PARTIDA (1 ${ }^{\text {a }}$ ETAPA).}

El Campus universitario de Reina Mercedes está localizado en la ciudad de Sevilla, siendo su eje principal la Avenida de Reina Mercedes. La ubicación geográfica del campus se caracteriza por su integración con la ciudad y una amplia gama de diversos tipos de transportes para su acceso. La zona cuenta también con un gran número de servicios comerciales y de restauración asociado a la comunidad universitaria. El límite del campus universitario es una zona de paso de vehiculos al centro de la ciudad y de acceso al área metropolitana, ver Fig. (3).

En el primer bloque de datos se obtuvo la información relativa a la distribución de los estudiantes y trabajadores, mostrando un total de 14.384 estudiantes, 800 profesores y 150 miembros del personal de administración y servicios de personal, repartidos en 8 edificios, en dos turnos de mañana y tarde. Estos datos se extrajeron de la memoria anual de la Universidad de Sevilla [25].

Después se creó el modelo de red por el que las calles e intersecciones se han de transformar en un grafo dirigido de $\operatorname{arcos} y$ nodos, ver Fig. (4) abajo a la derecha.

En fichas previamente diseñadas se realizó la captura de datos, teniendo como base la digitalización hecha por zonas. La informa-

ción se estructuró en los siguientes apartados:

- Información sobre las infraestructuras para la bicicleta: carriles, aparcamientos públicos y privados (dentro y fuera del campus universitario), ocupación de aparcamientos en diferentes horarios. El servicio público de bicicletas (Sevici) posee una página web de la cual se extrae el número de estaciones de aparcamientos de bicicletas en la vía pública, así como su ocupación.

- Información sobre el transporte público (autobús): ubicación de parada, rutas de líneas, horarios, frecuencias de paso, tarifas, información por tramos horarios de descenso y subidas de los usuarios por parada. La información fue proporcionada por la empresa de transportes publico Tussam.

- Información sobre la infraestructura de aparcamientos de vehículos: públicos, privados, de residentes, dentro del campus para los trabajadores y estudiantes y aforo de vehículos mal estacionados.

- Información de las infraestructuras para la movilidad peatonal: características y tipología del acerado y paso de peatones, flujos de paso de peatones.

- Infraestructuras para el vehículo privado: datos de la red viaria, número y tipo de carriles en cada vía, flujo de coches por hora (según el tipo de vehículo), movimientos o flujos en las intersecciones (giros), identificación de las vías principales de acceso a la zona en estudio. Parte de esta información

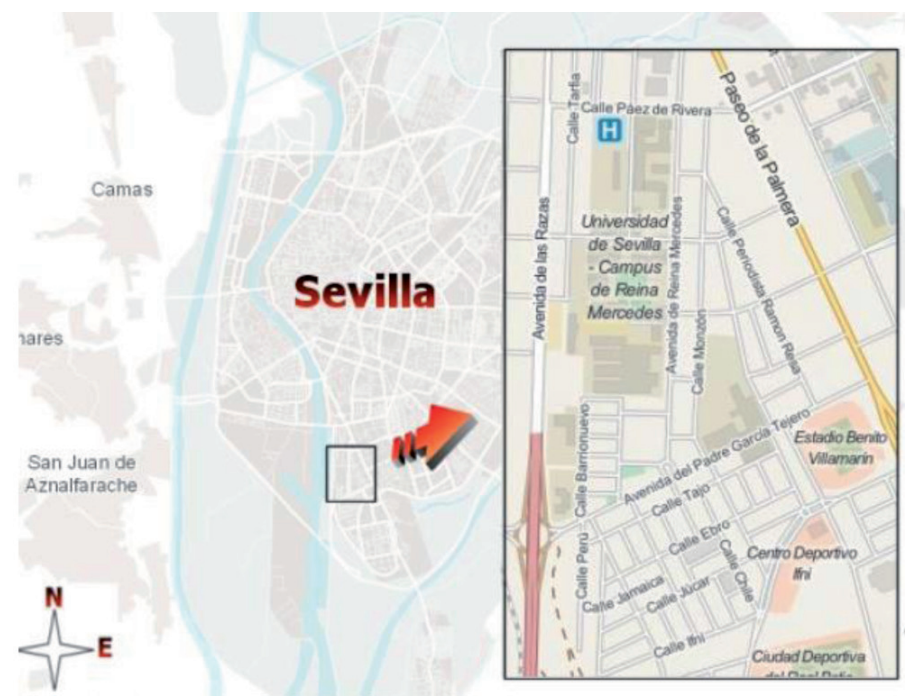

Fig. 3: Localización de la zona de estudio 


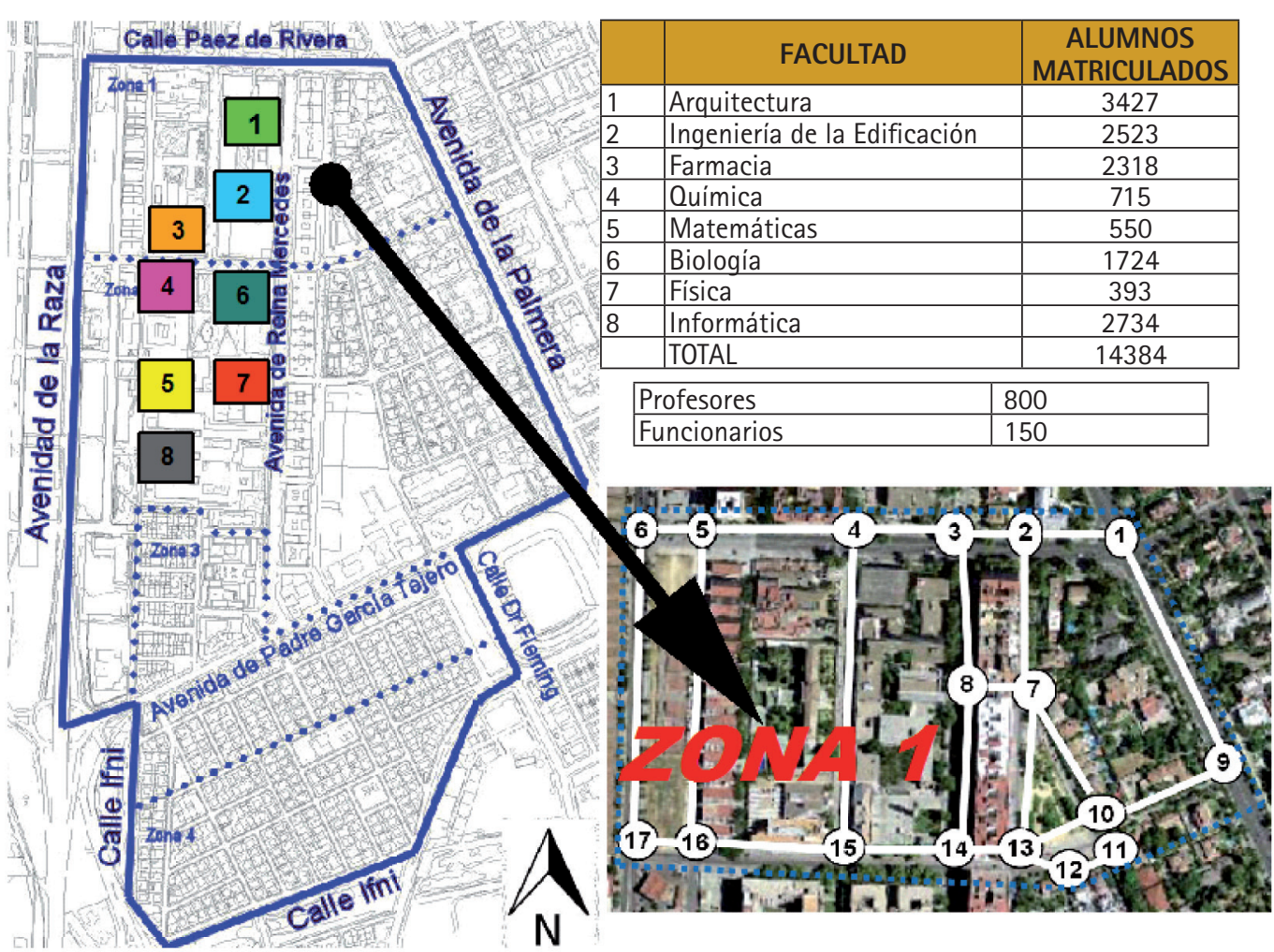

Fig. 4: Imagen de la zona objeto de estudio, con la localización de los centros, tabla de alumnos matriculados por centro y personal, y ejemplo de asignación de nodos en la zona 1 fue facilitada por el Área de Movilidad del Ayuntamiento de Sevilla.

En el segundo apartado de este punto, la realización de encuestas, el número a realizar fue distribuido por centros docentes con un nivel de confianza del $95 \%$ y una precisión del $90 \%$ y se muestra en la Tabla 2.

\begin{tabular}{|l|c|c|}
\hline \multicolumn{1}{|c|}{ FACULTAD } & $\begin{array}{c}\text { ALUMNOS } \\
\text { MATRICULADOS }\end{array}$ & $\begin{array}{c}\text { TAMAÑO DE } \\
\text { MUESTRA }\end{array}$ \\
\hline Biología & 1724 & 48 \\
\hline Farmacia & 2318 & 48 \\
\hline Fisica & 393 & 44 \\
\hline Matemáticas & 550 & 45 \\
\hline Quimica & 715 & 46 \\
\hline Arquitectura & 3427 & 48 \\
\hline ETSII & 2734 & 48 \\
\hline $\begin{array}{l}\text { Ingeniería de } \\
\text { Edificación }\end{array}$ & 2523 & 49 \\
\hline TOTAL & 14384 & \\
\hline
\end{tabular}

Tabla 2: Recomendaciones sobre tamaño muestral para encuestas de movilidad

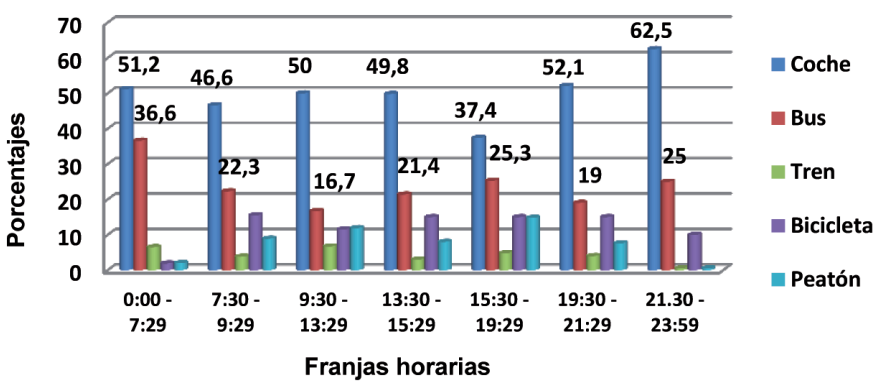

Fig. 5: Porcentaje de desplazamientos por medio de transporte y franja horaria por el menor número de autobuses. Por otra parte en las horas centrales aumentan los desplazamientos en bicicleta y peatón, debido principalmente a que la climatología de esta región favorece la utilización de estos medios y una mayor seguridad, aunque su uso está muy por debajo del medio predominante, el vehículo privado con un 50\% de uso medio, ver Fig. (5).

A través de las encuestas se obtuvo el tiempo empleado en cada medio de transporte. Su análisis puso de manifiesto que el peatonal es el modo con tiempos de viajes más bajo (por su cercanía al destino), seguido de la bicicleta y los modos mecanizados (comparables entre sí por tener longitud de desplazamiento muy similar en el ámbito urbano), ver Fig. (6). Otra característica que se puede observar es la estabilidad de los tiempos de viaje de los modos no mecanizados frente al vehículo privado con independencia de la hora del día. Esta característica es un factor positivo para describir actuaciones de cambio de modo basándose en la estabilidad de los tiempos de viaje.

\subsection{ANÁLISIS DE APARCAMIENTO}

El análisis de aparcamiento en el campus se basa en la relación entre el número de estudiantes y las plazas de aparcamiento

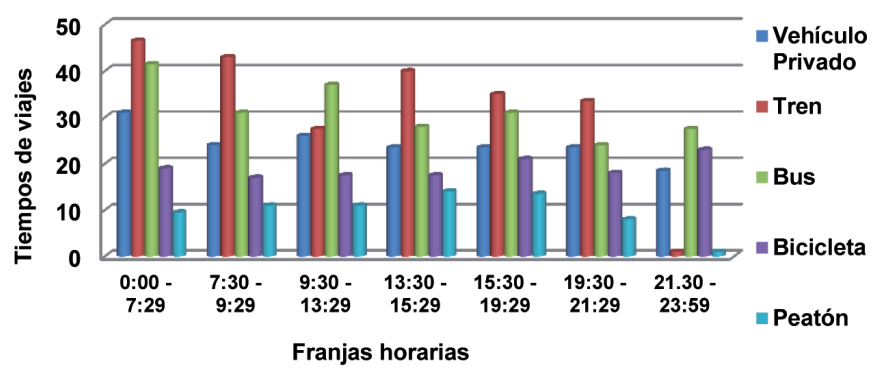

Fig. 6: Tiempos de viaje empleado, según el modo y franja horaria 
existentes. Esta información fue obtenida a través de la captura de datos en calle y se procesó en base a dos indicadores:

$\left(R_{a p, u}\right)$ : Ratio de plazas de aparcamiento del campus por estudiante. Ecuación (3).

$R_{a p, u}=\frac{N{ }^{\circ} a p u}{N{ }_{a l}}=\frac{590}{14384}=0,041$ plazas por alumno

$\left(R_{a p, t}\right)$ : Ratio de plazas de aparcamiento por estudiante en toda la zona de estudio. Ecuación (4).

0,29 plazas por alumno

$R_{a p, t}=\frac{N^{\circ} a p t}{N^{\circ} a l}=\frac{4186}{14384}=0,29$ plazas por alumno

Dónde:

"No apu" representa el número de plazas de aparcamiento disponibles dentro del campus.

"No ap" es el número total de plazas aparcamientos de toda la zona de estudio.

"No al" es el número total de alumnos.

Analizando el lugar donde los alumnos aparcan destaca que el $34 \%$ de los alumnos que usan vehículo privado encuentran una plaza de aparcamiento de las habilitadas por la universidad, mientras que el 63\% aparcan en las calles adyacentes o cercanas al centro o facultad de destino.
El tiempo medio de aparcamiento es una medida que sirve como referencia del nivel de competitividad de los modos alternativos con el vehículo privado. Un mayor tiempo de aparcamiento provoca un aumento del tiempo de viaje y hace a modos como la bicicleta o el transporte público más competitivos. Los datos sobre tiempo medio de búsqueda de aparcamiento en el campus indican que los usuarios del vehículo privado tardan una media de entre 10 y 15 minutos. El análisis diferenciado por zonas denota una mayor ocupación en la zona 1 (Facultad de Arquitectura), por la lejanía de los aparcamientos universitarios y de la existencia de otros centros atractores como la Dirección General de Tráfico y un centro hospitalario de tamaño medio. Así mismo también la zona 3 y 4 (más alejadas de los centros universitarios) se convierte en una zona de aparcamientos totalmente saturada, como consecuencia de la saturación de las zonas más cercanas a los centros (zona 1 y zona 2), ver Fig. (7).

La permisibilidad en la ilegalidad en el aparcamiento es un factor que influye sobre el uso del vehículo privado. El análisis de la ilegalidad por zonas mide el porcentaje vehículos aparcados ilegalmente frente a vehículos aparcados. Las zonas 1 y 2 , al igual que ocurre en la ocupación, son las que presenta mayor ilegalidad por su proximidad a los centros (ver Tabla 3).

La universidad dispone de 5 zonas de aparcamiento privadas dirigidas a los trabajadores y estudiantes con un total de 590 plazas de aparcamiento. Los niveles de ocupación indican que es máximo durante la mañana y disminuye significativamente por

\begin{tabular}{|c|c|c|c|c|c|}
\hline ZONA & No TOTAL VEHÍCULOS APARCADOS & No DE ILEGALES & \% ILEGALIDAD & No TOTAL DE APARCAMIENTOS & PLAZAS NO OCUPADAS \\
\hline 1 & 1205 & 145 & $12 \%$ & 1088 & 28 \\
\hline 2 & 1566 & 216 & $13,70 \%$ & 1429 & 79 \\
\hline 3 Y 4 & 863 & 35 & $4 \%$ & 875 & 47 \\
\hline TOTAL & 3634 & 396 & $10 \%$ & 3392 & 154 \\
\hline
\end{tabular}

Tabla 3: llegalidad de aparcamientos por zonas

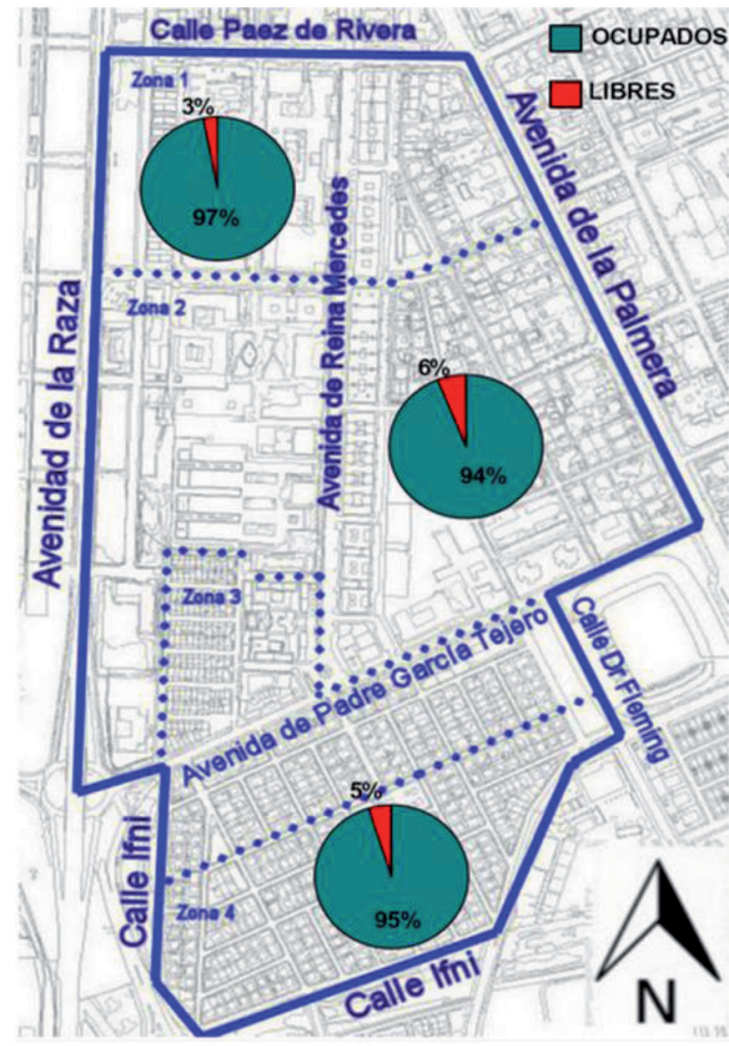

Fig. 7: Nivel de ocupación de aparcamientos distribuido por zona la tarde (50\%). Se observa que el 2,2 \% de las plazas que están libres por la mañana, están reservadas a personas con movilidad reducida, ver Fig. (8).

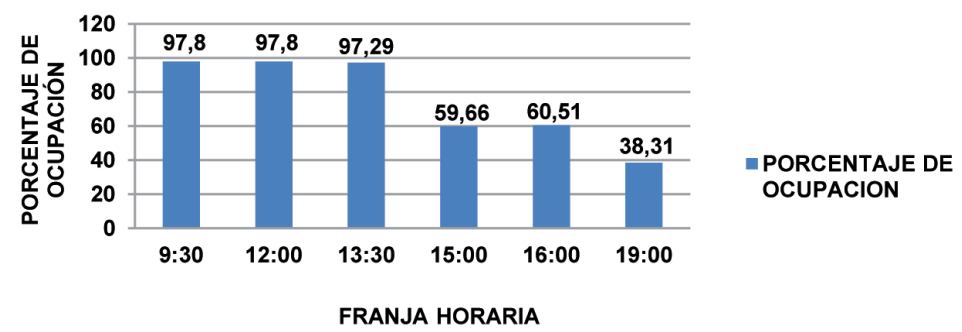

Fig. 8: Nivel de ocupación de aparcamiento privado del campus universitario

\subsection{ANÁLISIS DE LA CAPACIDAD DE MEJORA DE LA SOSTENIBILIDAD}

La capacidad de mejora en criterios de sostenibilidad se mide en la disponibilidad de los usuarios para cambiar a un modo de transporte más sostenible (transporte público, bicicletas o peatón) o reducir el uso del vehículo privado aumentando su ocupación (compartir coche). Del análisis de las encuestas se recoge esta disponibilidad pues se extrae que un $80 \%$ de los usuarios está dispuesto a compartir un viaje en un vehículo privado, siendo el $75 \%$ por ciento el conjunto de las personas que no utilizan el transporte público, ver Tabla 4. 


\begin{tabular}{|l|c|c|}
\hline \multicolumn{1}{|c|}{ FACULTAD } & $\begin{array}{c}\text { No uso de Transporte } \\
\text { Público (\%) }\end{array}$ & Compartir coche (\%) \\
\hline Arquitectura & 72,5 & 82,5 \\
\hline $\begin{array}{l}\text { Ing. de la } \\
\text { Edificación }\end{array}$ & 70 & 82 \\
\hline Farmacia & 78 & 88 \\
\hline Química & 80 & 78 \\
\hline Matemáticas & 72,5 & 80 \\
\hline Biología & 77,5 & 73,75 \\
\hline Física & 70 & 80 \\
\hline Informática & 83,5 & 81,25 \\
\hline MEDIA & $\mathbf{7 5 , 5}$ & $\mathbf{8 0 , 6 9}$ \\
\hline
\end{tabular}

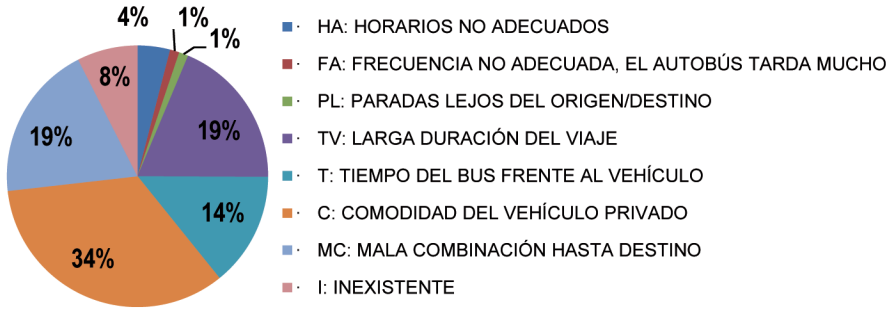

Fig. 9: Causas de no utilización del transporte público

línea 34 de bus en laFig. (10).

El indicador que representa el nivel de servicio de la línea en Tabla 4: Análisis de la no utilización de un transporte sostenible y predisposición a compartir vehículo privado

la zona viene expresado por la comparación del número de plazas

Analizado este $75 \%$ que no utiliza el transporte público se comprueba que los motivos principales son la comodidad del vehículo privado frente al transporte público (un 34\%) y el elevado tiempo de los viajes en el transporte públicos (un 19\%), ver Fig. (9).

Una de las necesidades a desarrollar en el campus son las medidas que fomenten el uso del transporte público y que permitan aumentar los niveles de ocupación en el vehículo privado.

4.3.1. Análisis del transporte público

El análisis del nivel de servicio de transporte público se realiza con objeto de estudiar si existe la necesidad de aumentar la capacidad de servicio. Para ello, se analizan las subidas y bajadas de viajeros en las paradas con independencias de las líneas, con estos datos se realiza un diagnóstico detallado de cada una de las líneas.

Para el análisis, por cada línea se diseña una ficha donde se recoge las principales características, como su longitud, número de paradas totales, paradas en la zona objeto de estudio y la distancia entre paradas. Cada línea se divide en dos secciones, la sección 1 tiene como destino el campus universitario y la sección 2 tiene como origen el campus universitario. Los principales indicadores obtenidos en la ficha proporcionan información sobre el nivel de saturación (oferta de la línea frente a demanda), frecuencia de paso de las líneas, y la distribución del número de subidas y bajadas de pasajeros por paradas e intervalos horarios, ver ejemplo de la ficha para la

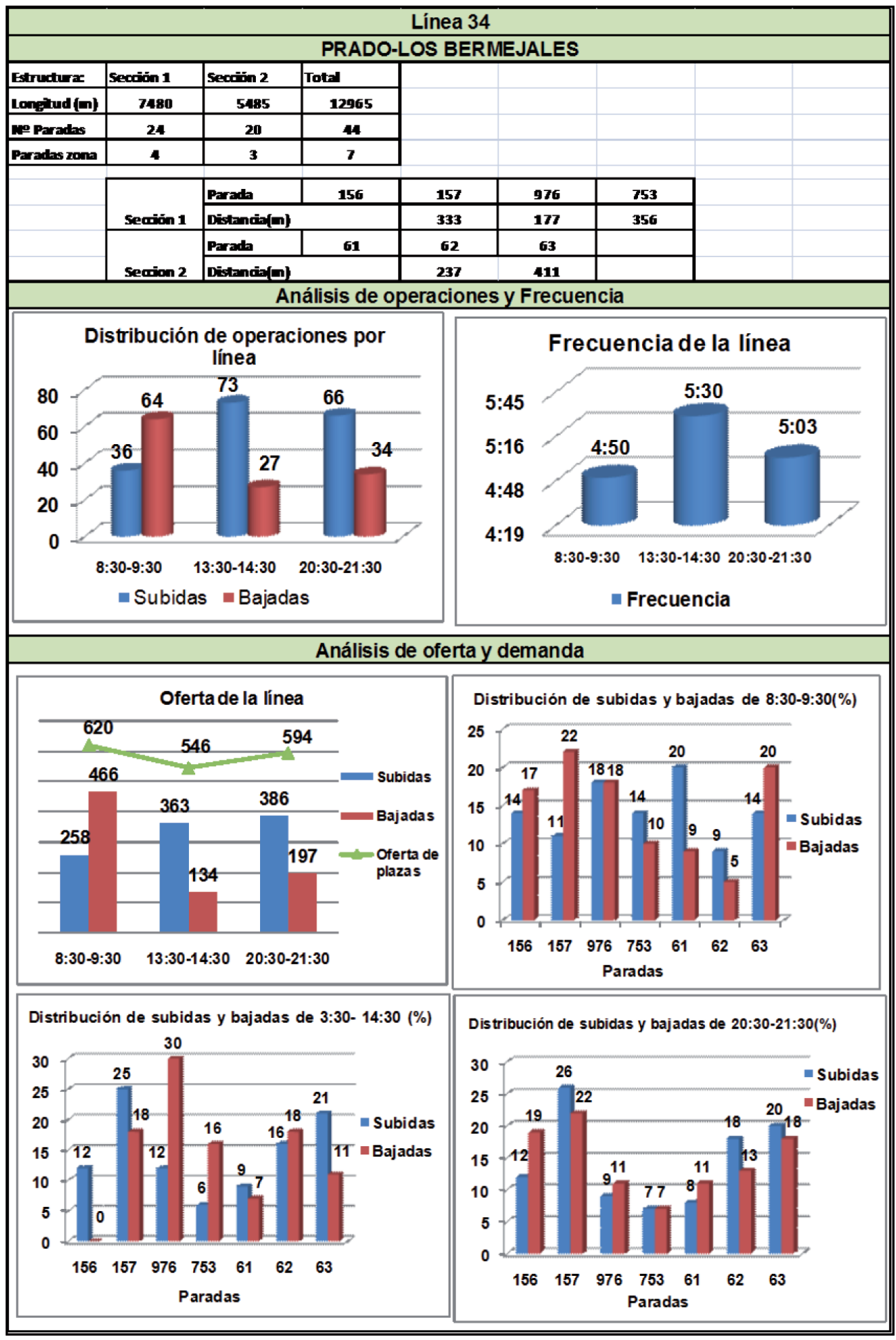

Fig. 10: Ficha resume del análisis de la línea 34 de autobús 
Personas subidas y bajadas

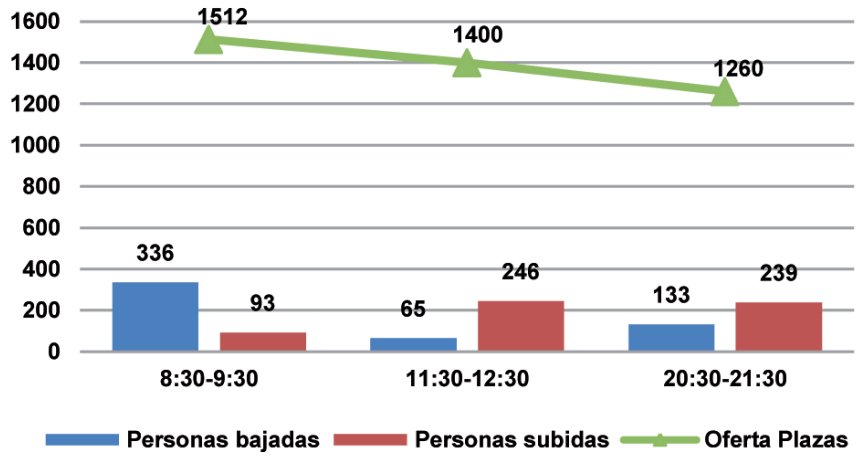

Fig. 11: Análisis comparativo entre el nivel de servicio y la demanda

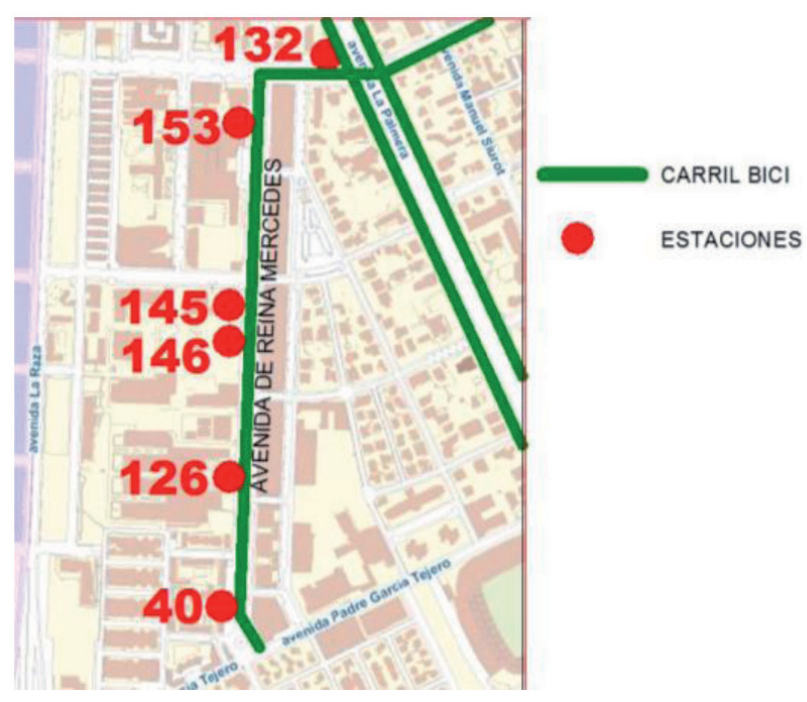

Figura 12: Disposición de estaciones de Sevici en Reina Mercedes

ofertadas y nivel de ocupación (oferta de la línea). Fig. (11). Este indicador permite analizar la necesidad de aumentar la capacidad del sistema de transporte público y en el presente estudio se ha fijado este valor a un 70\% (la relación entre oferta y ocupación), que corresponde con el nivel máximo de comodidad en un desplazamiento en transporte público.

En el análisis comparativo entre la oferta y la demanda (índice de servicio) se puede observar como el número de plazas disponibles, en diferentes horarios, es muy superior a la demanda.

\subsubsection{Análisis de la movilidad ciclista}

El análisis de la movilidad ciclista se basa en conocer la evolución del servicio público de bicicletas, la movilidad ciclista privada y el nivel de ocupación de aparcamiento de bicicletas.

El área dispone de seis estaciones con una oferta de 144 bicicletas o ranuras para estacionamientos del servicio público de bicicletas (Sevici) según la Oficina de Accesibilidad de la Gerencia de Urbanismo del Ayuntamiento de Sevilla. Fig. (12).

La Universidad dispone de un convenio con el propio Ayuntamiento para el uso del servicio por parte de los alumnos. La Tabla 5 muestra el número de abonados a dicho servicio.

\begin{tabular}{|c|c|c|c|}
\hline Facultad & SEVICI & Alumnos & Usuarios/Total \\
\hline Arquitectura & 1585 & 3427 & $45.73 \%$ \\
\hline Ing. de Edificación & 837 & 2523 & $33.39 \%$ \\
\hline Farmacia & 681 & 2318 & $30.28 \%$ \\
\hline Química & 214 & 715 & $31.38 \%$ \\
\hline Matemáticas & 131 & 550 & $26.95 \%$ \\
\hline Bilogía & 528 & 1724 & $31.81 \%$ \\
\hline Física & 123 & 393 & $36.72 \%$ \\
\hline Informática & 1009 & 2734 & $32.96 \%$ \\
\hline TOTAL & 5108 & 14384 & $33.09 \%$ \\
\hline
\end{tabular}

Tabla 5: Abonados al sistema público de bicicletas Sevici

La distribución de la utilización por centro está influida por la proximidad de estaciones y aparcamientos de bicicleta. El indicador de distribución de uso por centros refleja la influencia de la proximidad de aparcamiento para bicicletas privadas y estaciones de bicicleta pública, en la Fig. (13) se muestran los porcentajes de uso de la bicicleta pública, particular y ambas por centro.

En el análisis del uso de los tipos de bicicleta cabe destacar la definición del indicador de distribución del uso de las bicicletas por titularidad. Este tipo de indicador permite analizar la influencia de la distancia de las estaciones públicas en el uso del servicio. Fig. (14).

Al igual que en el transporte público, se define un indicador del nivel de saturación del sistema público de bicicletas. El indicador desagrega en el tiempo (un día) la disponibilidad de bicicletas y

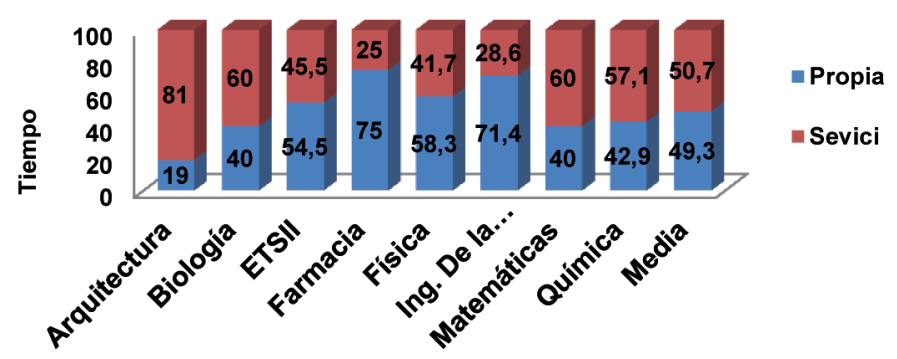

Fig. 14: Porcentaje de uso de la bicicleta pública (Sevici) y privada

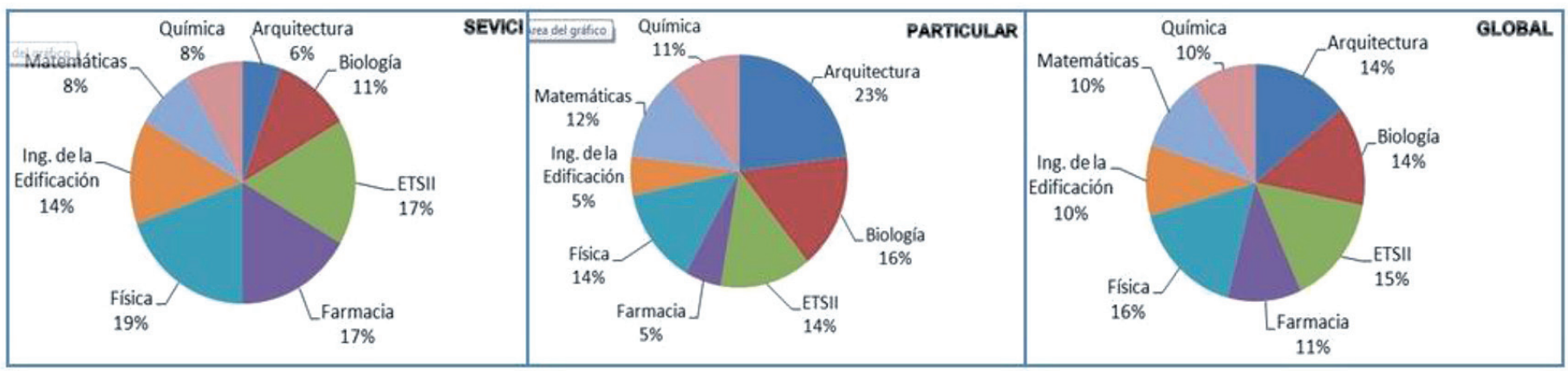

Fig. 13: Porcentajes de uso de la bicicleta pública, particular y en global por centro 
aparcamiento. Esto permite determinar en qué instante o intervalo horario el sistema queda saturado ya sea por falta de bicicletas o

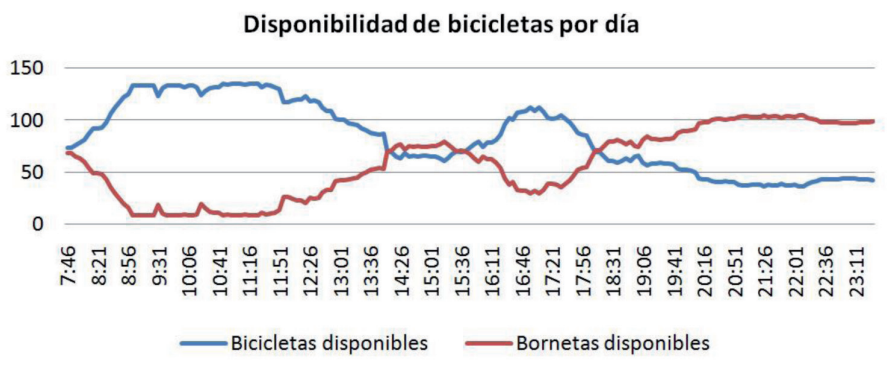

Fig. 15: Evolución diaria de la disponibilidad de bicicletas públicas

por la no disponibilidad de aparcamiento. Fig. (15).

El análisis por estación se estructura mediante fichas que recopilan la evolución de la estación en cuanto a la disponibilidad de bicicletas y bornetas durante un periodo de tiempo que corresponde desde las 7:30 de la mañana a las 12 de la noche, las tasas de llegadas y salidas de bicicletas a lo largo del día, los niveles de ocupación y un resumen del nivel de ocupación media diaria. En el presente estudio se ha fijado a un 5\% el máximo de saturación (bornetas o bicicletas disponibles) para proponer medidas de mejora del servicio.

La principal consecuencia del análisis del horario del servicio público de bicicletas denota que las mañanas (9:00 - 12:30) suponen un punto crítico donde no se dispone de bornetas, es decir, el sistema queda saturado. A partir de las 12:30, coincidiendo con el fin de clases en el nuevo horario de grado (8:30 a 12:30), la disponibilidad de bornetas aumenta. Finalmente se analizaron las causas para la no utilización de la bicicleta, cuyos resultados son reflejados en la Fig. (16).

Visto que una de las problemáticas a la hora de analizar las estaciones de bicicletas es la no disponibilidad de bicicletas o bornetas en horas críticas, una de las soluciones que se propone es que la Universidad dentro del propio campus instale un centro de gestión integral para el almacenamiento de bicicletas que permita retirar o reponer bicicletas en los momentos de saturación.

\section{INVENTARIO ENERGÉTICO Y AMBIENTAL. 3a ETAPA}

Como punto final del diagnóstico se estima el inventario energético y ambiental de los desplazamientos al campus universitario. Los resultados obtenidos se estructuran por modo, tipo de contaminante y consumo de combustible, esto se traduce a su equivalente en tonelada de petróleo que permite estimar el coste energético incurrido. Tabla 6.

El inventario energético y ambiental obtenido es empleado

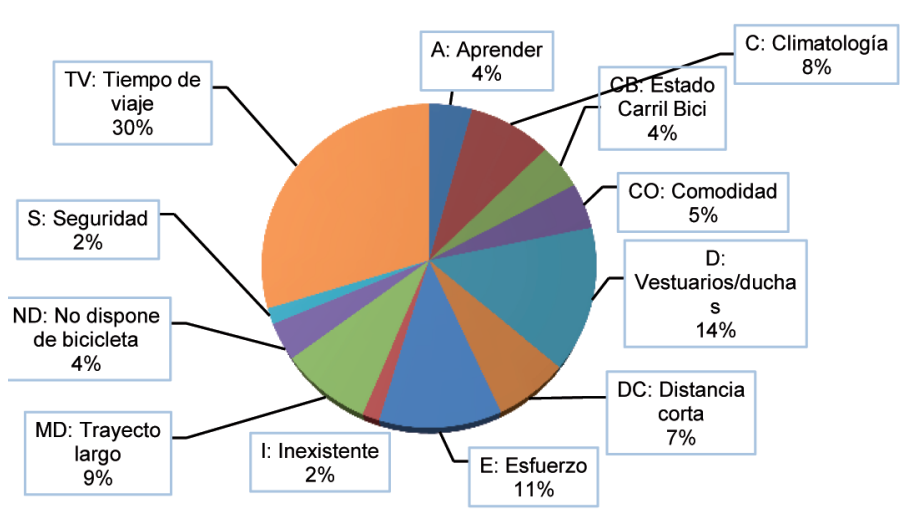

Figura 16: Causas de la no utilización de la bicicleta

como un referente más para incentivar las propuestas de mejoras, es decir, las actuaciones que se realicen o propongan se basarán también en la mejora de estos resultados, teniendo como objetivo final la disminución del consumo y las emisiones.

Los factores de conversión empleados en el inventario energético y ambiental se basan en los proporcionados por la Agencia Andaluza de la Energía (Directrices del IPCC, Intergubernamental Panel on Climate Change).

Como resultado final de los cálculos, una vez extraídos de las encuestas el modo de transporte, distancia y ocupación, obtenemos los datos de consumo según el modo de transportes y expuestos en la Tabla 6.

Analizando los datos del $\mathrm{CO}_{2}$ en la Fig. (17) se observa que el vehiculó privado es el modo de transporte con mayor carga de emisión con diferencia, seguido a gran distancia por el transporte

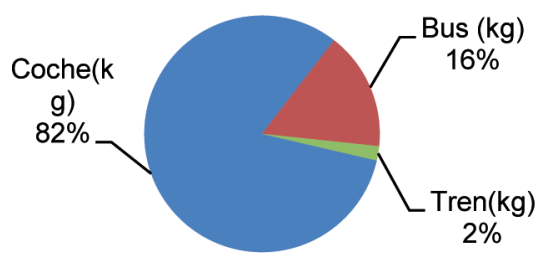

Fig. 17: Distribución de emisiones de CO2 por modos de transporte y día

público.

Desagregando el inventario por centro obtenemos los datos de la Tabla (7).

Analizando los datos obtenidos para $\mathrm{Co}_{2}$ mediante gráfica obtenemos el reparto expuesto en la Fig. (18).

En proporción con el número de alumnos es la facultad de Arquitectura la que tiene un mayor nivel de emisiones de $\mathrm{CO} 2$. Esta facultad está ubicada en la zona 1 que es la más saturada en

\begin{tabular}{|l|c|c|c|c|c|c|c|c|c|}
\hline & $\begin{array}{l}\text { Inventario } \\
\text { Ambiental }\end{array}$ & \multicolumn{2}{|c|}{} & \multicolumn{5}{c|}{ Inventario Energético } \\
\hline & & \multicolumn{2}{|c|}{ Litros } & \multicolumn{3}{|c|}{ Tep $^{(1)}$} & & \\
\hline & Co2 & Co & Hc & NOx & Gasolina & Gasóleo & Gasolina & Gasóleo & Total \\
\hline Coche(kg) & 960,185 & 84,016 & 11,602 & 6,001 & 168,737 & 211,426 & 0,135 & 0,185 & 0,320 \\
\hline Bus $(\mathrm{kg})$ & 189,675 & 2,710 & 1,355 & 2,439 & 37,457 & 49,486 & 0,034 & 0,039 & 0,073 \\
\hline Tren $(\mathrm{kg})$ & 22,863 & 0,001 & 0,001 & 0,071 & 0,000 & 0,108 & 0,000 & 0,000 & 0,000 \\
\hline Toneladas(por $\mathrm{km})$ & 1,173 & 0,087 & 0,013 & 0,009 & 206,194 & 261,020 & 0,169 & 0,224 & 0,394 \\
\hline Total Toneladas & 14,483 & 1,047 & 0,159 & 0,108 & 2568,438 & 3267,929 & 2,120 & 2,797 & 4,916 \\
\hline
\end{tabular}

(1)Toneladas equivalentes de petróleo

Tabla 6: Inventario ambiental por modo de transporte y día 


\begin{tabular}{|l|c|c|c|c|c|c|c|c|c|}
\hline & $\begin{array}{c}\text { Inventario } \\
\text { Ambiental } \\
\text { (Toneladas) }\end{array}$ & & & & & \multicolumn{3}{c|}{ Inventario Energético } \\
& & \multicolumn{2}{|c|}{ Litros } & \multicolumn{3}{c|}{ Tep } & & \\
\hline & Co2 & Co & Hc & N0x & $\begin{array}{c}\text { Gasolina/ } \\
\text { Gas }\end{array}$ & Gasóleo & $\begin{array}{c}\text { Gasolina/ } \\
\text { Gas }\end{array}$ & Gasóleo & Total \\
\hline Arquitectura & 4,543 & 0,345 & 0,051 & 0,033 & 810,583 & 1025,332 & 0,665 & 0,881 & 1,546 \\
\hline Biología & 1,679 & 0,124 & 0,018 & 0,012 & 293,230 & 372,737 & 0,241 & 0,320 & 0,561 \\
\hline ETSII & 2,794 & 0,185 & 0,029 & 0,022 & 499,770 & 637,673 & 0,417 & 0,541 & 0,958 \\
\hline Farmacia & 1,283 & 0,096 & 0,014 & 0,009 & 225,413 & 286,282 & 0,185 & 0,246 & 0,431 \\
\hline Física & 0,423 & 0,031 & 0,005 & 0,003 & 74,330 & 95,507 & 0,061 & 0,082 & 0,143 \\
\hline Ing. de la Edificación & 2,461 & 0,170 & 0,027 & 0,019 & 440,051 & 560,113 & 0,366 & 0,477 & 0,842 \\
\hline Matemáticas & 0,457 & 0,032 & 0,005 & 0,003 & 74,956 & 100,387 & 0,061 & 0,086 & 0,148 \\
\hline Química & 0,843 & 0,065 & 0,010 & 0,006 & 150,106 & 189,899 & 0,123 & 0,163 & 0,286 \\
\hline Total Campus & 14,483 & 1,047 & 0,159 & 0,108 & 2568,438 & 3267,929 & 2,120 & 2,797 & 4,916 \\
\hline
\end{tabular}

Tabla 7: Inventario ambiental por centro y día

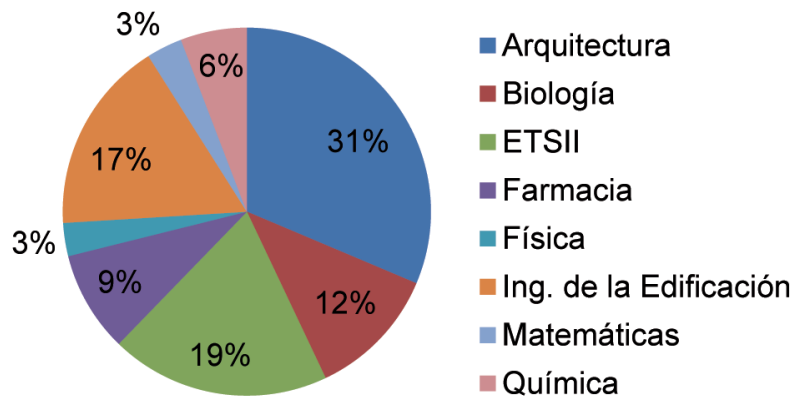

Fig. 18: Distribución de emisiones de CO2 por centro y día

aparcamientos (comparando la dimensión real de ésta con las demás zonas). Por otra parte, esta facultad es la más cercana a otros centros atractores como la Dirección General de Tráfico y el centro hospitalario ya mencionado.

Destacar que este consumo diario de combustible, de 4,9 Tep (Toneladas equivalentes de petróleo), arrojan una cantidad de 14,5 toneladas de $\mathrm{CO}_{2}$ a la atmosfera y esto solo correspondiente al movimiento de un campus universitario de 14.384 alumnos integrado en una ciudad de 700.000 habitantes.

Teniendo en cuenta que los viajes a campus o centros universitarios suponen hasta un $10 \%$ de los desplazamientos realizados dentro de la ciudad, se estarían emitiendo hasta 145 toneladas diarias de $\mathrm{CO}_{2}$ a la atmosfera de la ciudad.

\section{PROPUESTAS DE ACTUACIÓN. 4ª ETAPA}

Una vez analizados los resultados obtenidos se proponen una serie de medidas en diferentes ámbitos:

- Potenciar el uso del servicio público de bicicletas, ya que es unos de los métodos más efectivos para reducir el uso del vehículo privado. La evolución del uso de la bicicleta ha ido aumentando, pasando de un reparto modal de un $5 \%$ en el año 2007 a 8,9\% en el año 2012. Según un 69\% de los usuarios, los principales motivos del aumento de su uso son que es un medio más económico, dispone de un menor tiempo de desplazamiento y es de fácil aparcamiento [26].

El fomento del uso del servicio público de bicicletas debe estar acompañado de un incremento de la disponibilidad de bornetas y bicicletas en determinados horarios (Figura 15). En horas punta de llegada existe falta de bornetas libres e igualmente a la hora de salida faltan bicicletas, por lo que se ve la necesidad de instalar más estaciones e igualmente gestionar tanto la retirada en horas punta de llegada con la reposición en horas punta de salida, para ello sería muy conveniente la instalación de un centro de gestión de la bicicleta ubicado en el campus. Para fomentar su uso y satisfacer estas necesidades se están estableciendo convenios entre Sevici y el Ayuntamiento de Sevilla.

- Reducción del número de plazas de aparcamientos en la vía pública, con ello se evita el efecto llamada del vehículo privado e igualmente gestionar las plazas existentes para los vehículos con mayor ocupación.

- La potenciación de los transportes colectivos. A través de los indicadores se mostró que la capacidad del servicio es superior a la demanda, por lo que es necesario potenciar aún más su uso, para ello una de las medidas principalmente utilizadas son la reducción de su coste o incentivos al colectivo universitario. El Servicio de Transporte Público ha puesto en marcha una campaña en la que el colectivo universitario se puede beneficiar de un Carnet anual de transporte público con un precio de un 34\% inferior al anterior, el cual ha tenido muy buena aceptación en este colectivo.

- Concienciación al colectivo sobre los beneficios personales y a la comunidad del uso de transportes más sostenibles para la reducción de contaminantes emitidos a la atmosfera. Existe una falta de información y concienciación en el campus, sería necesario incorporar programas de información relacionadas con la movilidad y herramientas que potencien la sostenibilidad, como por ejemplo, el desarrollo de aplicaciones para compartir coche, información sobre transportes alternativos o incluso cesión temporal de bicicletas.

\section{CONCLUSIONES}

En este artículo se presenta un análisis de movilidad a un campus universitario en la ciudad de Sevilla, introduciendo herramientas para el cálculo del consumo energético y emisiones de contaminantes ambientales, que sirven como indicadores para el análisis de la movilidad y que se pueden implementar fácilmente en cualquier estudio de transporte sostenible como consecuencia de la movilidad urbana en una zona determinada. De esta forma la palabra sostenible cobra un mayor significado al contemplar 
estos parámetros ambientales y obtener una cuantificación sobre el nivel de contaminación provocado, que normalmente no se contemplan en este tipo trabajos. Esto permite tener un mayor nivel de concienciación sobre el daño producido al medio ambiente.

El diagnóstico realizado ha seguido un proceso de 4 fases. En el análisis de estas, se detecta que para el aumento del nivel de demanda en el transporte público se han de llevar a cabo políticas y acuerdos, como el conseguido con el carnet universitario para el uso anual de los estudiantes. Igualmente para el aumento del uso de la bicicleta pública, se ha de llegar también a acuerdos con la universidad, para la gestión de los puntos de estacionamientos en horas de mayor demanda, así como la disminución de tarifas para estudiantes.

La fase de propuestas de actuación pone de manifiesto que es necesario la aplicación de medidas coercitivas en el campus universitario de Sevilla para desalentar el uso del vehículo privado, como podría ser la aplicación de un coste o tarifa para el uso de aparcamiento del vehículo privado y aumentar la capacidad del servicio de bicicleta pública.

Destacar que para el desarrollo de los métodos del análisis de la movilidad y del transporte, el análisis energético y ambiental es una base de conocimiento para el seguimiento de las actuaciones futuras y debería ser tenido en cuenta por los organismos públicos.

Por último, comentar que el Libro Verde "Hacia una nueva cultura de la movilidad urbana" de la Comisión Europea reconoce la movilidad urbana como un factor importante de crecimiento y empleo, con un fuerte impacto en el desarrollo sostenible. También pone en relieve la dificultad de conciliar el desarrollo económico de las ciudades para obtener una mejor calidad de vida y de protección del medio ambiente.

\section{BIBLIOGRAFÍA}

[1] Baucire F. 2007. La mobilité au centre de I'urbain, Compte rendu de la journée de la d'étude sur la mobilité et développement urbain organisée par l'ADP.

[2] Robusté Francesc. Plan de movilidad sostenible de los campus Norte y Sur de la UPC. 2011. Centro para el desarrollo Técnico Industrial (CENIT).

[3] Rozas José Fermín. 2009. Movilidad sostenible en el Campus Miguel de Unamuno de la Universidad de Salamanca. Congreso Nacional de Medio Ambiente, Conama 9.

[4] Toor Will, 2004. Transportation \& Sustainable Campus Communities. Issues, Examples, Solutions. ISBN: 1-55963-922-9.

[5] Colomer José,2010 Plan de transporte para la Universidad Politécnica de Valencia: Campus de Alcoy Instituto de transporte y territorio. Universidad de Valencia.

[6] Es por Madrid. Cálculo del coste de utilización del vehículo privado(http://www.espormadrid.es/2011/10/una-herramienta-para-calcular-el. html?utm_source=twitterfeed\&tutm_medium=twitter\&tutm_campaign=Fe ed\%3A+espormadrid+\%28es+por+madrid\%29, último acceso enero 2015).

[7] Ministerio de Fomento. Estrategia Española de Movilidad Sostenible -(http://www.fomento.gob.es/NR/rdonlyres/149186F7-0EDB-4991-93DDCFB76DD85CD1/46435/EstrategiaMovilidadSostenible.pdf; último acceso Marzo 2105)

[8] Guía práctica PTT para la elaboración e implantación de planes de transporte al centro de trabajo (IDAE).

[9] Dirección General de Tráfico. Parque de vehículos por tipos, provincias y carburantes -(http://www.dgt.es/portal/es/seguridad_vial/estadistica/ parque_vehiculos/por_provincia_tipo_y_carburante/ última visita, enero 2015).

[10] IDAE. 2006. "PMUS: Guía práctica para la elaboración de planes de movilidad urbana sostenible." Isbn-13: 978-84-86850-98-2 Isbn-10: 84-86850-98-3.

[11] IPCC, 2007. Cambio climático 2007: Informe de sintesis. Cuarto Informe de evaluación del Grupo Intergubernamental de Expertos sobre el Cambio Climático [Equipo de redacción principal: Pachauri,R.K. y Reisinger, A. (directores de la publicación)]. IPCC, Ginebra, Suiza.

[12] Ibeas, A. Gonzalez, F. Dell'Olio, L. Moura, J.L. 2007. Manual de encuestas de movilidad. Preferencias reveladas unversidad de Cantabria ISBN 978-84-

10 | Dyna | Marzo - Abril 2016 | Vol. 91 n² | 0/10
89627-73-4.

[13] Plan de energías renovables 2005-2010. Consejo de ministros del 25 de Agosto de 2005

[14] Racero Moreno, Jesús, Eguía Salinas, Ignacio, Guerrero López, Fernando; Bada Tomas, Manuel; Escamez Berenguer, Jesús. 2012. Técnicas y herramientas de evaluación de estrategias para la movilidad sostenible.

[15] Linee Guida per la redazione del piano urbano della mobilità di area vasta del comne di Venecia e del territorio dei comuni appartenenti all'area centrale della procinzia de Venecia. [Ven09] Linee Guida per la redazione del piano urbano della mobilità di area vasta del comne di Venecia e del territorio dei comuni appartenenti all'area centrale della procinzia de Venecia (PU

[16] Guía de los planes de locales de transportes del Reino Unido. LPTs - $\quad$ http://www.dft.gov.uk/pgr/regional/ltp/guidance/fltp/ agoodpracticeguideforthedeve3656 último acceso enero 2015.

[17] Plan de déplacements Urbains Francia. www.stif-idf.fr último acceso enero 2015

[18] Racero Moreno, Jesús, Eguía Salinas, Ignacio, Guerrero López, Fernando. 2008. Emission Inventory for Urban Transport in the Rush Hour: Application to Seville. En: Air Pollution. Pag. 291-300.

[19] Juan de Dios Ortuzar, Luis G. Willonson. 2002. "Modelling Transport." Editorial Wiley. ISBN 0471861103.

[20] Racero Moreno Jesús, Hernández, Marta, Guerrero López, Fernando, Racero,Gregorio. 2011. An integrated support system and a GIS tool for sustainable transportation plans. Vol. 3. Pag. 355-372. En: Information Technologies in Environmental Engineering. ISBN 978-3-642-19536-5.

[21] Balsas, C. J. 2003. Sustainable transportation planning on college campuses. Transport Policy, 10(1), 35-49.

[22] Miralles-Guasch, C., \& Domene, E. 2010. Sustainable transport challenges in a suburban university: The case of the Autonomous University of Barcelona. Transport policy, 17(6), 454-463.

[23] Shannon, T., Giles-Corti, B., Pikora, T., Bulsara, M., Shilton, T., \& Bull, F. 2006. Active commuting in a university setting: assessing commuting habits and potential for modal change. Transport Policy, 13(3), 240-253.

[24] AEMA. 2014. "Inventarios Nacionales de Emisiones a la Atmósfera" Documento resumen.

[25]Anuario estadístico 2010-11. Universidad de Sevilla. "servicio.us.es/secgral". [26] Fuente investigación sobre el uso de la bicicleta. Sibus. 2011. 